\title{
ALFABETIZACIÓN EN LA COMUNIDAD RURAL DE AMÉRICA LATINA: LAS NIÑAS EN LA ESCUELA ${ }^{1}$
}

\author{
Silvia-Maria Chireac \\ Universidad de Valencia \\ Norbert Francis \\ Northern Arizona University (EEUU)
}

\begin{abstract}
RESUMEN: El siguiente informe analiza los resultados de tres estudios de caso en dos escuelas rurales de México y una de Ecuador. Comparamos los índices de paridad de género (IPG) fijando el análisis en dos dimensiones: la inscripción/asistencia y el desempeño en la alfabetización. Los resultados generaron una hipótesis tentativa que sugiere un marco conceptual para una propuesta de investigación más amplia. Proponemos, con base en la evidencia de estudios previos de un importante avance en el IPG en la región latinoamericana, la evaluación del aprovechamiento escolar en la lectoescritura en escuelas primarias rurales que enfoca la comparación entre niños y niñas. Retomando como modelo los procedimientos de los tres estudios de caso del presente informe, los objetivos de la evaluación independiente serían: (1) comprobar o rechazar el estimado oficial de la paridad (100/100) en el acceso a la primaria en las zonas rurales, y (2) comprobar o rechazar la hipótesis que ha surgido de los tres estudios preliminares - que para las niñas, una vez matriculadas, el desempeño verificable (por medio de una prueba de comprensión de lectura) resulta estadísticamente equivalente, o superior, en comparación con el desempeño de los niños, hasta en las comunidades apartadas y de escasos recursos materiales.
\end{abstract}

PALABRAS CLAVE: Escuelas rurales, alfabetización, niñas, América Latina.

1. Agradecemos a Galo Rodrigo Guerrero-Jiménez, Norman Alberto González Tamayo, María de los Ángeles Guamán, Ángel Martínez de Lara, Carlos María Vacacela Medina y Bertha María Villalta Córdova de la Universidad Técnica Particular de Loja (Ecuador) por su colaboración en la implementación de las evaluaciones en la comunidad de San Lucas, Loja, y por sus observaciones sobre la primera versión de las pruebas de lectoescritura, y el apoyo de la UTPL a través del proyecto: Bilingüismo e interculturalidad, aprendizaje de segunda lengua y aprovechamiento escolar en la comunidad de San Lucas, provincia de Loja. 


\title{
LITERACY LEARNING IN THE LATIN AMERICAN RURAL COMMUNITY: GIRLS IN SCHOOL
}

\begin{abstract}
The following report analyzes the results of three case studies in rural schools, two from Mexico and one from Ecuador. Indices of gender parity (IGP) were compared directing the analysis toward two dimensions: inscription/attendance and actual performance in literacy learning. The results generated a tentative hypothesis that in turn suggests a conceptual framework for a proposal for further research on a larger scale. We propose, based on evidence from previous studies showing important advances in IGP in the Latin American region, the assessment of school achievement in the area of literacy in rural primary schools focusing on the comparison between boys and girls. Taking as a model the assessment methods of the three case studies, the objective of the of independent evaluation would be the following: (1) confirm or reject the official estimate of parity (100/100) in regard to access to rural primary school, and (2) confirm or reject the hypothesis of the present preliminary studies in Mexico and Ecuador - that for girls, once registered in school, their verifiable progress (measured by a test of reading) will be shown to be statistically equivalent, or superior, in comparison to the progress of boys, even in marginalized communities of limited material resources.
\end{abstract}

KEYWORDS: Rural schools, literacy learning, girls, Latin America.

Recibido: 29/03/2017

Aceptado: 17/07/2017

Correspondencia: Silvia-Maria Chireac, Universidad de Valencia, Departamento de Didáctica de la Lengua y la Literatura, Av. dels Tarongers, 4, 46022 Valencia. Email: Silvia.Chireac@uv.es.

\section{INTRODUCCIÓN}

A partir del año 1990, la investigación educativa en América Latina ha podido concentrar recursos importantes en las comunidades fuera de los centros urbanos, en particular con un enfoque sobre el estudio de los procesos de aprendizaje en el aula. Surge de una toma de conciencia entre investigadores, que la discusión de los problemas en las ciencias de la educación debe basarse cada vez más en el análisis de datos empíricos y del trabajo de campo. Hamel $(2008,2009)$ es un ejemplo destacado, entre otros; consiste en iniciativas importantes sobre la evaluación sistemática del desempeño real en el ámbito lingüístico, tomando en cuenta el factor del bilingüismo en el aprendizaje de la lectura y la escritura. En paralelo, la UNESCO ha registrado las tendencias a nivel regional desglosando los resultados por país, en particular el avance en cada caso en el acceso a la escolaridad. El presente estudio retoma una dimensión de esta investigación: la paridad de género respecto al acceso a 
los servicios educativos, y aparte, respecto al desempeño académico, las habilidades ligadas a la alfabetización por parte de niños y niñas. En poco tiempo cumpliremos treinta años de este trabajo en las regiones rurales. Durante este periodo, la UNESCO (2015) ha marcado un cambio importante: en la región, en conjunto, en la escuela primaria, secundaria y media superior, el sistema escolar ha logrado, según los informes oficiales, una paridad (menos de un punto de diferencia) entre niñas y niños. Por cada 100 de los primeros se inscriben 100 de las segundas (100/100 se traduce en un índice de 1.00, el Índice de Paridad de Género, IPG). En los dos países en que hemos realizado estudios, México y Ecuador, se reporta un IPG de 1.00, la paridad completa (inscripción/asistencia).

Todavía quedan desafíos y pendientes urgentes en relación con la desigualdad de oportunidades, el acceso efectivo por parte de las poblaciones desfavorecidas, la eficiencia terminal (problema de la deserción), en las zonas de pobreza persistente, y en las mismas regiones desfavorecidas, la falta de paridad (a pesar de los avances desde 1990) en los niveles superiores (Hall y Patrinos, 2004; Atal et al., 2009). Los desequilibrios documentados, en efecto, motivaron el presente análisis del aprovechamiento académico en tres comunidades, de diferentes condiciones culturales y socioeconómicas, que presentaremos a continuación.

Las comunidades rurales deben hacer frente a múltiples desafíos a pesar de los avances que se han ido produciendo a lo largo de los años en varios ámbitos. Primero, entre todos los avances debemos destacar la expansión significativa del sistema escolar, junto con el acceso al aula para sectores de la niñez antes excluidos, detalle que abordaremos en las siguientes secciones. En relación con la igualdad de oportunidades en la educación siguen persistiendo obstáculos que se percatan todavía en la falta de acceso universal a la escuela y a los materiales de enseñanza. La primera pregunta que planteamos para la región latinoamericana es: si todavía persiste un desequilibrio entre niñas y niños respecto al acceso a estos recursos, en el contexto de la no-universalidad en zona rural (siempre tomando en cuenta la paridad en inscripción). Luego, reconociendo la persistencia de la brecha en el IPG para los niveles superiores en zonas apartadas y de bajos recursos - desventaja para las niñas (Vezzali, 2016) - ¿cómo podemos caracterizar el desequilibrio? ¿En qué, precisamente, consiste?

Aunque en la Cumbre Mundial de las Naciones Unidas celebrada en 2005 se hizo hincapié en eliminar la desigualdad y el desequilibrio de género con el objetivo de garantizar a las niñas un acceso equitativo a una educación básica de buena calidad, así como un buen rendimiento académico, parece evidente que la situación sigue en estado latente y se está tardando en alcanzar dicho objetivo en los municipios rurales (Céspedes y Robles, 2016). Además, Berlinski et al. (2009) y Meyers (2004) señalan la etapa preescolar como fundamental para adquirir las competencias básicas que se requieren para cursar con éxito las asignaturas académicas de la primaria y la secundaria. A nivel mundial, si comparamos los datos del año 1999 en la enseñanza primaria sobre la presencia de las niñas en la escuela, percibimos que había 92 niñas escolarizadas por cada 100 niños (UNESCO, 2015). Con el paso del tiempo, la disparidad de género ha disminuido considerablemente hasta llegar a una tasa de 97 niñas en el año 2012. Para el año 2017, en América Latina, podemos destacar el logro, histórico por su alcance en condiciones difíciles en varios países todavía (p. ej., la no-universalidad de inscripción y la deserción en algunos sectores), de una 
paridad, en matrícula neta, entre niñas y niños, en la escuela primaria, secundaria y media superior de 100/100. Sin embargo, en el campo el abandono de estudios persiste como factor limitante serio. Según un estimado de la UNICEF (2015: 23) "en los estratos más pobres 12 de cada 100 niños y niñas no concluyen la primaria, mientras que solamente 2 de cada 100 niños y niñas de los estratos de más alto ingreso no la culminan. Las tasas de conclusión de primaria son de 96 por ciento en zonas urbanas contra 85 por ciento en zonas rurales".

Históricamente, la deserción o la no inscripción pesaban con más fuerza sobre las niñas de las zonas rurales que, muchas veces, comprometían sus estudios a favor de las tareas domésticas y el cuidado de hermanos menores. También la distancia entre la escuela y sus hogares, la falta de medios de transporte, los gastos derivados de la escolarización, como libros y uniformes, y la prohibición de las adolescentes embarazadas o casadas de asistir a la escuela sumaban a otras prácticas discriminatorias. La segunda pregunta que presentamos se relaciona también con la diferencia en el IPG entre zonas urbanas y rurales: ¿han surgido condiciones favorables (para las niñas) en la primaria rural para revertir la tendencia de abandonar los estudios antes del sexto año, que, por ejemplo, todavía no se han materializado en los niveles superiores? Cabe preguntarnos por qué el informe de la UNESCO (2015) indica que en algunos países la tasa de permanencia de las niñas hasta finales de la primaria ha mejorado y hasta ha superado la de los niños. En los niveles superiores, en las zonas marginadas, los casos de disparidad en la escolarización persisten en detrimento de las niñas. ¿Se trata de factores negativos que tienen su raíz en la primaria, que son propios de los niveles superiores, o se deben a una confluencia de factores que persisten a pesar de los avances en la participación femenina en la primera? Y por fin, preguntamos: ¿llegando a las comunidades más apartadas y marginadas de América Latina, también podemos comprobar, en los hechos, el mismo índice de IPG en primaria (matrícula - 1.00) que los Ministerios de Educación marcan a nivel nacional? Además, ¿es la paridad efectiva (en términos medibles), en el aprovechamiento académico?

Antes de presentar los resultados de las evaluaciones y las entrevistas de México y Ecuador, Ilamamos la atención a los resultados de la prueba PISA, instrumento que evalúa el rendimiento de los alumnos de 15 años a nivel mundial, en escuelas urbanas y rurales. Una brecha interesante que se ha presentado por años y de manera uniforme de un país al otro aparece entre la lectura y las matemáticas. En la lectura, las niñas logran mejores resultados que los niños (UNESCO, 2012). En cambio, en la prueba de matemáticas, los niños son los que obtienen mejores calificaciones. Desafortunadamente, en nuestro estudio, solo pudimos tomar una muestra de las habilidades de lectoescritura. No obstante, la relación reportada por la prueba PISA queda como referente internacional importante ${ }^{2}$.

2. Dejamos a los especialistas en el desarrollo de las habilidades matemáticas escolares la evaluación de este importante aspecto del conocimiento infantil. El ámbito del proyecto, así como la competencia de los investigadores, se limitaban al campo de la lingüística aplicada. La investigación centró su trabajo en la adquisición de la primera lengua, el aprendizaje de segundas lenguas y la lectoescritura. En todo caso, tomando en cuenta los resultados de PISA, empezar por una comparación en el área de la lectoescritura nos permite plantear una expectativa: un desempeño comparable entre niños y niñas, no superior por la parte femenina. En ningún caso esperamos (según nuestra hipótesis) un resultado distinto. 
Unterhalter et al. (2014) elaboraron una teoría del cambio (TdC) que enfatiza la afirmación de que la educación de las niñas y la igualdad de género se ven afectadas por procesos que ocurren dentro y fuera de la escuela. Según los autores, hay tres tipos de interacciones que se deben combinar con el objetivo de mejorar la escolaridad de las niñas: (i) las que reúnen los recursos y la infraestructura, (ii) las que se centran en el cambio institucional, y finalmente, (iii) las que proporcionan el cambio de normas y la inclusión de los grupos marginados en la toma de decisiones educativas.

Siguiendo los ejes proporcionados por la $\mathrm{TdC}$ en relación con las intervenciones centradas en los recursos y la infraestructura, una prioridad consiste en minimizar el efecto de los factores económicos y sociales que obstaculizan la asistencia y la participación (p. ej., favorecer la cercanía de los centros escolares que abarcan los niveles superiores, secundaria y bachillerato). Hoy en día los prejuicios y las resistencias culturales pesan mucho menos, pero todavía es necesario atenderlos. Asimismo, en lo que concierne las reformas al sistema educativo, se debería contar con profesores bien formados en diferentes niveles del sistema educativo, sobre todo en relación con las actitudes de género y la capacitación para saber abordar explícitamente la igualdad de género en sus propias prácticas de enseñanza, aprendizaje y gestión, lo cual revertiría en la elaboración de los materiales de aprendizaje y las prácticas pedagógicas fundamentales para garantizar la igualdad de oportunidades. Finalmente, otra prioridad clave para mejorar la escolarización de las niñas en las zonas rurales tiene que ver con las intervenciones para cambiar las normas de género y aumentar la participación de las mujeres en el sistema educativo. Con el objetivo de mejorar la escolarización de las niñas, se deberían establecer interconexiones y lazos dinámicos entre las tres áreas de intervención.

Con este marco de fondo, presentamos tres estudios de caso en comunidades rurales de México y Ecuador sobre la participación de las niñas en la primaria (dos casos) y la secundaria (uno). En particular, nos centramos en el proceso de la alfabetización escolar, comparando su experiencia de aprendizaje con la de sus compañeros varones. Los resultados se limitaron a la descripción, partiendo de estudios exploratorios en cada localidad; sugirieren la presentación de una propuesta de investigación, con una predicción concreta. El marco de fondo en los tres casos también cuenta, hoy en día, con una circunstancia importante, por toda la evidencia que nos fue disponible: el libre acceso a las instalaciones y la inscripción abierta para las niñas de estas comunidades. Al parecer, refleja no solo la política educativa del sistema escolar, sino también un cambio histórico en las expectativas culturales en las mismas comunidades. Al mismo tiempo, queremos enfatizar que mientras las tres comunidades comparten la característica de ser rurales, no proponemos ninguna confrontación entre las calificaciones, entre los diferentes programas educativos, ni una comparación o un contraste a lo largo del tiempo entre el primer estudio, realizado en durante el año escolar 1991-1992, y los segundos, 2010 y 2016, respectivamente. La comparación, informal (que le encargamos al lector), radica entre las descripciones en cada caso, por separado, y las tendencias regionales reportadas en esta sección por las investigaciones internacionales de la UNESCO e instancias afines. El análisis de los resultados generará una hipótesis de trabajo que presentaremos en la conclusión como propuesta para un proyecto de investigación regional en América Latina. 


\section{Alfabetización escolar en Tlaxcala, Chiapas (México) y Loja (Ecuador)}

\section{Tlaxcala}

Nuestro estudio en el poblado de San Isidro, municipio de Vicente Guerrero, localizado en la región náhuatl-hablante en los altos del sur del estado, comunicado por carretera con el gran centro urbano de Puebla (Luna Ruiz, 2007; Nava Nava, 2014), abarca un periodo de veinticinco años. El seguimiento de observaciones sobre la cuestión del acceso femenino a la escuela primaria nos permitió dar constancia de cambios radicales, a partir del año 1991. De reciente inauguración, las autoridades educativas estatales introdujeron un programa bilingüe con la sustitución de la plantilla por maestros que podían impartir las clases en las dos lenguas. Regresamos a la sección sobre análisis y reflexión en cuanto a la consideración del factor bilingüe, la incorporación de la primera lengua de los estudiantes en la instrucción. La iniciativa fue acompañada de una expansión gradual en los servicios escolares para corregir una inscripción del 75\% entre niños de edad escolar, la tasa más baja del estado, en la comunidad con la tasa más alta de analfabetismo (INEGI, 1990). Al mismo tiempo, junto con el incremento general en la asistencia, se registró, con los años, un ingreso femenino cada vez mayor. Aun así, en aquel año, pudimos registrar una tendencia a la deserción: en los grados $1^{\circ}$ hasta $4^{\circ}$, un IPG acercándose a 1.00, pero con disminución sensible en $6^{\circ}$.

No obstante, los resultados de la batería de pruebas lingüísticas revelaron una paridad global notable respecto al desarrollo de las habilidades de lectoescritura (comprobada por separado en los tres grupos bajo estudio): en una muestra de 45 alumnos, dividida entre $2^{\circ}, 4^{\circ}$ y $6^{\circ}$, las evaluaciones bilingües de comprensión de lectura, redacción y resumen oral arrojaron promedios sorprendentemente comparables (Figura 1). Por comodidad, como se nota en la figura, sumamos las calificaciones en español y en náhuatl; pero constatamos que en español académico (meta principal de la instrucción) las niñas y los niños sacaron promedios estadísticamente equivalentes en las tres evaluaciones. Retomando la Figura 1, resumimos el diseño de la evaluación: Comprensión de lectura -promedio de dos medidas (análisis cualitativo de errores en lectura oral y prueba cloze con el apoyo de una serie de palabras), Redacción- una evaluación de la expresión escrita apoyada con la presentación de un modelo (de género narrativo) y apoyo contextual en forma de tres ilustraciones; Resumen oral es una medida de compresión complementaria en donde el niño rememora el texto que acaba de leer. La misma distribución (ninguna diferencia significativa) se evidenció en un estudio sobre la conciencia metalingüística $(\mathrm{CM})$ : reflexión sobre las propiedades formales de palabras en español y náhuatl. La prueba de CM califica la habilidad de identificar la lengua, su "origen" (español o náhuatl), en la que están escritas palabras (una lista de 25 vocablos) que forman parte del léxico bilingüe de los hablantes de la comunidad (por ejemplo, XICOHTENCATL ¿es español o náhuatl?). Para un análisis y resumen de los procedimientos y los resultados, ver Chireac et al. (2017a) y Francis (2012).

Así que aún antes del gran auge en las inscripciones, paralelas a las tendencias marcadas a nivel regional por la UNESCO, tomamos nota de un aprovechamiento en la lectoescritura uniforme entre niños y niñas. Tomando en cuenta la totalidad de las 


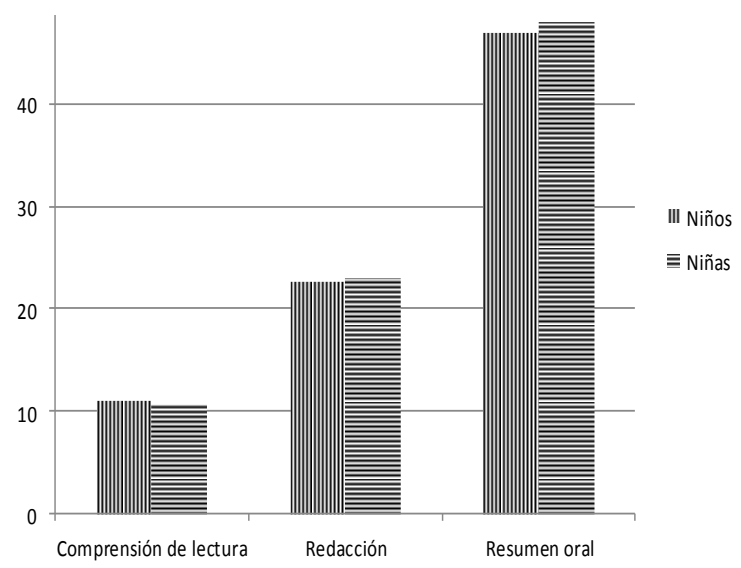

Figura 1. Lectoescritura bilingüe (español + náhuatl): segundo, cuarto y sexto grado

evaluaciones y entrevistas, en solo una medida se pudo marcar una diferencia notable entre niñas y niños: en la categoría de percepciones y creencias relacionadas con el uso del lenguaje, por separado en cada lengua, la llamada Entrevista Sociolingüística $(E S){ }^{3}$ A la pregunta si recordaban en ocasiones cuando les daba pena hablar en voz alta en la presencia de otras personas, los varones hacían memoria de la sensación con más frecuencia que sus compañeras de clase, tanto en español como en náhuatl. Pero, al consultar a los informantes locales del proyecto, optamos por no analizar las respuestas (p. ej., como índice de "lealtad sociolingüística") más allá de reportarlas. Para las otras preguntas relacionadas con las percepciones y creencias no surgieron diferencias significativas (p. ej., ¿cuando seas grande, qué lengua, o lenguas, les vas a enseñar a tus hijos?). En suma, sólo en una evaluación no-académica pudimos diferenciar entre niños y niñas; y aun así quedó inconclusa la interpretación.

Veinticinco años después, de visita a la misma escuela primaria, pudimos comprobar la paridad entre los graduados del sexto año, primer avance en evidencia sobre el desequilibrio de los años 90s. Mientras en 1991 muy pocas jóvenes se des-

3. La Entrevista Sociolingüística tomó una muestra de percepciones y criterios afectivos sobre el uso del lenguaje de los mismos 45 niños de los grados $2^{\circ}, 4^{\circ}$ y $6^{\circ}$ que se presentaron para las pruebas de lectoescritura y conocimiento bilingüe. Contestaron a preguntas en formato de entrevista individual, en privado (aproximadamente 30 minutos, alcanzando una respuesta completa a las preguntas por parte de todos los participantes) en las siguientes categorías:

- identificación de la lengua (español o náhuatl) de una serie de textos (pares, en traducción, de un cuento, letrero, recado...);

- juzgar la "utilidad" entre los pares español-náhuatl (cual es mejor para contar, poner un letrero, dejar un recado...);

- saber el nombre de las dos lenguas que hablan;

- recordar una ocasión cuando le dio pena hablar en público;

- qué lengua hablan con: el maestro, amigos, padres, abuelos;

- cuál le gusta más;

- en conversación con los amigos, cuál es más fácil;

- es posible que algún día el náhuatl desaparezca en San Isidro, y cómo se sentirían;

- ¿cuando sea grande, qué lengua, o lenguas, le va a enseñar a sus hijos? 
plazaban para estudiar en la secundaria o la preparatoria más cercana, hoy aproximadamente el 25\%-30\% de la inscripción es femenina y cursan materias en el nuevo Centro Escolar, igual que en el recién inaugurado CECyTE ${ }^{4}$ del pueblo vecino ${ }^{5}$. Entre las niñas de la muestra original de 1991 (22 entre 45), solo una niña llegó a terminar la preparatoria. Los testimonios de su historia quedan reflejados en sus comentarios sobre las vicisitudes por las que pasó en una entrevista que nos concedió hace dos años. Precisamente, durante este periodo, notamos una tendencia importante: hace cincuenta años, no poder hablar la lengua nacional se asociaba con una condición social caracterizada por la segregación y la marginación. Ya no se da esta asociación con tanta fuerza por la sencilla razón de que el sector de la población monolingüe en lengua indígena ha disminuido notablemente (INEGI, 2010). A diferencia de hace una generación, las madres de esta primera generación de bachilleras dominan el español casi por completo (todavía, hoy, a diferencia de sus abuelas). En otras palabras, ya no se evidencia entre las mujeres menores de cuarenta años un vínculo entre el conocimiento del español y condición social.

Para confrontar estos avances con las condiciones del siglo pasado, principio de los años 1960s, remitimos al lector al estudio antropológico más completo de la región (Nutini y Isaacs, 1974). Su registro etnográfico presenta el panorama de una comunidad de aislamiento y extrema pobreza, de una minoría de los hogares que poseían camas, sillas o utensilios de metal; la mayoría de las casas contaban con pisos de tierra. Así como en las aldeas circunvecinas, observaron un alto nivel de monolingüismo en lengua náhuatl, por la mayor parte entre las mujeres. Casi "toda la gente [andaba] descalza". No había mercado en la comunidad, y a pesar de su cercanía, muy pocos de los hombres buscaban trabajo en Puebla (p. 89).

\section{Chiapas}

La segunda investigación que presentamos parte de un estudio llevado a cabo por nuestro colega Oscar Chanona Pérez en el año 2010 en la escuela primaria "Niños Héroes" de la comunidad lacandona de Lacanha Chansayab, situada en una región desfavorecida del estado de Chiapas, el más pobre de México (Levy et al., 2016). Actualmente el centro escolar de educación básica destaca por la presencia de un número mayor de niñas en comparación con los varones que hace más de 10 años; incluso hay más niñas que finalizan el ciclo de primaria y muchas de ellas optan por continuar la educación secundaria. Hasta perciben, según una de las informantes, la urgencia, en mayor grado que los jóvenes varones, de perfeccionar su español y aprender a leer y escribir con mayor destreza. La decisión de las niñas de seguir estudiando resulta patente en el contexto lacandono, puesto que ellas mismas consideran que por un lado, los estudios les pueden ayudar a salir de la po-

4. Colegio de Estudios Científicos y Tecnológicos - nivel bachillerato.

5. Estimados, en total, que $25-30 \%$ de la inscripción en el Centro Escolar y el CECyTE era femenina por medio de la observación en dos visitas a las instalaciones, y una prueba piloto de lectura que se administró a los estudiantes. Se trata de cientos de niñas en la comunidad, de nuevo ingreso a los niveles superiores, secundaria y media superior. En la ceremonia de graduación de Primaria, los estudiantes se enfilaban por separado, niños y niñas, facilitando el cálculo y la comprobación de paridad. En la entrevista con la directora, se comprobó el dato. 
breza al tener la oportunidad de estar inmersas socialmente en la vida productiva y por otro lado, afirman que una formación académica les da permiso a acceder más fácilmente a mejores condiciones de vida, a penetrar y posicionarse en ámbitos sociales en los que antes no eran aceptadas por analfabetismo.

El estudio pretende ofrecer una explicación sobre el impacto que tiene la escolarización sobre la identidad de las niñas lacadonas con base en una serie de entrevistas con las alumnas de la secundaria local en 2010. La alfabetización de las niñas en un contexto dominado exclusivamente por varones permite al colectivo femenino que se integre en la vida social gracias a la adquisición de destrezas lingüísticas y valores sociales que les ayudan a tener unas percepciones claras de los papeles que desempeñan en la sociedad junto a los adolescentes y a los hombres.

En resumen, las mujeres de Lacanha, las más pobres y excluidas del estado de Chiapas, pueden tener acceso a funciones distintas en la sociedad y sentirse parte integrante de la comunidad en la que viven, "lo que conlleva una reconfiguración de su identidad" (Chanona, 2014: 145) al adoptar elementos de otras culturas, pero sin dejar de preservar sus símbolos étnicos.

A continuación, citamos una muestra de los testimonios elocuentes y puntuales, registrados por el equipo de Chanona Pérez - cómo las entrevistadas entienden el cambio de generación entre la de las hijas y la de las madres:

[Le] ayudo a mi mamá para lavar la ropa, le ayudo a cobrar también y a dar cambio (2014: 162).

[Tampoco] sabe hacer bien las cuentas porque mi mamá nunca fue a la escuela... aprendió hablar español con sus hermanas pero no fue a la escuela y no puede escribir, no sabe.

Ahora hay telesecundaria aquí, antes tenían que ir más lejos y su papá no lo dejaba, por eso es que mi mamá ya no estudió más que la primaria. Es que su mamá de mi mamá tenía otra mentalidad (p. 158). [Me] dice: ...que no te quedes como yo, sólo vendiendo artesanía en Bonampak (p. 157).

Trabajo en el restauran con mi mamá, ayudo a hacer la comida, a pasar la comida en la mesa y también cobrar la cuenta porque mi mamá no sabe hacer bien la cuenta (p. 162).

Si estudio, ...puedo buscar trabajo, darle dinero a mi mamá, porque ella no tiene dinero y tiene que trabajar mucho, trabaja en la artesanía, en la casa y va a la milpa, y yo por eso quiero estudiar para ayudarle a ella y para que yo tenga otro trabajo, no como el de mi mamá, ir a otra ciudad y tener mi propio negocio, que sea mío. (p. 162)... trabajar con la computadora en una oficina (p. 163).

En efecto, según una de las informantes, la inscripción femenina, saliendo de la Primaria, en dos salones, es superior a la masculina: "[En] la secundaria somos 5 mujeres y 3 hombres"..."En mi salón...también somos más mujeres: 4 mujeres y 3 hombres" (p. 149).

\section{Loja}

El tercer estudio de caso proviene de la provincia sureña de Ecuador en su primera etapa de descripción etnográfica y diseño de instrumentos de evaluación, realizada 
en $2016^{6}$. Los niños de Primaria que participan en las pruebas exploratorias enfocadas en el aprovechamiento académico son de la etnia Saraguro, comunidad del cantón de San Lucas. Por su ubicación sobre la carretera Panamericana se encuentra más comunicada que las comunidades de Tlaxcala y Chiapas, pero no intentamos cuantificar este factor (la primera tampoco la caracterizaríamos de aislada y geográficamente distante). La única diferencia que marcamos, con certeza, se relaciona con el dominio de la lengua nacional: todos los alumnos de San Lucas, sin excepción, son hablantes nativos del español, ver King (2001) para el único estudio sociolingüístico reciente de la región disponible.

De una muestra total de 95 alumnos en los tres grados $\left(2^{\circ}: N=20,4^{\circ}: N=34\right.$, $6^{\circ}: \mathrm{N}=41$ ) pudimos pasar una prueba de comprensión de lectura (Chireac et al., 2017b) para determinar el nivel de confiabilidad aceptable. Entre otros patrones descriptivos, Ilama la atención notar a quiénes pertenecen las calificaciones superiores: en segundo año, el primer lugar, 14/20, lo ocupa una niña; en cuarto, 19/20, igual. En sexto, cuatro resultados son de la máxima puntuación, 20/20, todos ${ }^{7}$ de niñas. De igual manera como en los estudios de caso mexicanos, los resultados reflejan no solo el nuevo acceso igualitario a la escuela primaria, sino sugieren niveles de aprovechamiento comparables con sus pares varones (Figura 2); que, están aprovechando, valga la redundancia, unos aspectos, por lo menos, de su nueva integración, históricamente hablando, al sistema educativo. Son aspectos de la experiencia en el aula ligados al aprendizaje de las destrezas y habilidades que subyacen al uso productivo del lenguaje académico. Globalmente, los alumnos avanzan en su comprensión de lectura; la diferencia entre $2^{\circ}$ y $4^{\circ}$ resulta significativa, $t=5.57, p<.001$, así como entre $4^{\circ}$ y $6^{\circ}, t=5.31, p<.001$, tal como era de prever. Además, Ilama la atención el desempeño de las niñas en la misma materia, quienes mantienen una tendencia favorable frente a sus compañeros varones. Su ventaja de 1.35 puntos (16.07-14.72) no llega a ser estadísticamente significativa $(t=1.37, p=.180)$, pero resulta evidente que, académicamente, durante los años de la primaria se defienden con bastante soltura.

En el caso de San Lucas, destacamos diferencias significativas en la comparación entre segundo y sexto de primaria. Paralelamente a los resultados positivos en la evaluación de la lectura, salta a la vista una relación curiosa entre los 41 alumnos de sexto: una inclinación numérica en la asistencia a favor de las niñas, de 30 a 11.

6. La elaboración de las evaluaciones en Loja abarcó las habilidades de la lectoescritura en un intento de mejorar el diseño de la serie anterior, implementada en Tlaxcala, en parte con miras a una aplicación similar a la que estamos proponiendo en la conclusión del presente trabajo. La evaluación de la redacción quedó igual como estaba por haber cumplido con los requisitos de transparencia de aplicación y una robusta validez de resultados. Modificamos las pruebas de comprensión de lectura para: en primera instancia, introducir una variante de elección múltiple en la prueba cloze, utilizando textos de género narrativo, igual como en la versión anterior. La segunda modificación consiste en la presentación de textos que requieren la identificación y corrección, por separado, de errores de ortografía y gramática.

7. Un indicador metodológico importante apuntamos sobre el problema de la selección de los participantes, que varió entre los estudios de Tlaxcala y Loja. En el primero pedimos a los docentes que seleccionaran a los estudiantes que progresaban normalmente, y que en apariencia carecían de trastornos serios de aprendizaje (o de la vista - en 1991 ninguno de los niños en el pueblo Ilevaba gafas). En Loja, apartamos del presente análisis las pruebas de los alumnos que no llegaron a intentar por lo menos la mitad de los ítems, incluso después de ensayar el procedimiento en sesión de instrucción previa (eran nueve: 4 niñas, 5 niños, todos del segundo año). 


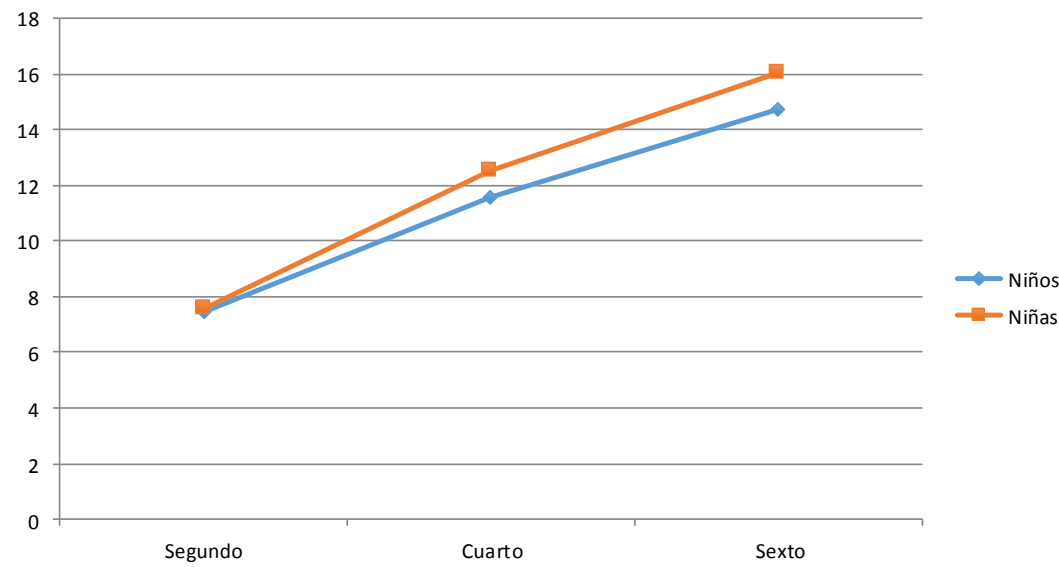

Figura 2. Compresión de lectura: español

Todavía no disponemos de testimonio o explicación por parte del personal docente por la diferencia; mínimamente, la calificamos de balance favorable. En segundo, hay 13 mujeres y 16 hombres; en cuarto, 17 y 17. Tampoco contamos, aún, del importantísimo dato sobre la inscripción/asistencia en los grados inferiores de la actual generación de los curso de sexto.

\section{ANÁLISIS Y REFLEXIÓN}

\section{Retrospectiva y estudio del contexto actual}

La relación entre los avances en la paridad académica y las tendencias sociales más amplias se presenta como objeto de estudio complementario. Como señaló el estudio de Chiapas, para un grupo de personas a quienes ha sido denegada la oportunidad, aprender a leer y escribir y aprender la lengua nacional se inscriben dentro de un proceso de profundos cambios de identidad. Vinculada con la tendencia que mencionamos sobre el incremento del bilingüismo entre las mujeres, es su participación más visible en las actividades económicas fuera del hogar, en el comercio por ejemplo, e incluso en empleos asalariados en la cabecera municipal o en la ciudad. Ofrecemos otro ejemplo. El proyecto en Tlaxcala colabora con un taller comunitario, recientemente establecido, de alfabetización y aprendizaje del español como segunda lengua. La mayoría de los integrantes del taller son señoras con hijos pequeños, más un señor. La coordinadora del taller recibió el año pasado su título de bachillerato por medio del mismo instituto de educación para adultos, INEA, del cual el taller forma parte. Como iniciativa propia e independiente, es la primera de su naturaleza en la comunidad, en particular respecto a su acercamiento bilingüe sobre el desafío de fomentar las habilidades expresivas. O sea, dichas habilidades, promovidas en la primera lengua, apoyan el desarrollo de la expresión en español, la segunda lengua. Se trata de mejorar la expresión verbal en general, y con el tiempo, en la expresión escrita. 
De esta manera, una de las hipótesis que podríamos explorar considera el factor del modelo educativo bilingüe para las comunidades autóctonas (política oficial de los gobiernos en los tres casos), donde es posible identificar implementaciones válidas. Un modelo de instrucción de menos exclusión (por no dificultar la comprensión de los contenidos escolares al sector monolingüe no-hispanohablante) favorecería, plausiblemente, a la población infantil femenina en las regiones más aparatadas y conservadoras (Chiodi y Bahamondes, 2002; Díaz Montenegro, 2012; INM, 2006). En Tlaxcala, por ejemplo, se dio una correlación entre la implementación del modelo bilingüe y la inscripción/asistencia femenina, pero no podemos afirmar que fue de causa/efecto. No disponemos de datos históricos más precisos sobre esta posibilidad (la de un factor-causa) en las tres regiones de nuestro estudio. La presentamos como propuesta de investigación para otras comunidades y regiones.

Por fin, nos permitimos especular sobre un factor de amplia incidencia tanto en México como en Ecuador: el avance en la democratización del sistema educativo en evidencia a todas luces, reflejo del paulatino proceso democrático nacional en los dos casos. Invitamos al lector a repasar los cambios políticos relevantes desde principios de los años 1990s hasta hoy en día.

En la presentación de los tres estudios de caso, es importante destacar que, en efecto, son casos; que no pretendemos ofrecerlos como representativos. Nos cayeron al azar en trabajos de campo en escuelas rurales en México y en el Ecuador. Por un lado, los datos que salieron de las muestras están compatibles con el nuevo acceso de las niñas a la primaria y la secundaria en las comunidades rurales, un logro general ya reconocido. Tal y como mencionamos en la Introducción, la pregunta que se presenta ahora respecto a la experiencia escolar de las niñas es si experimentan condiciones adecuadas de aprendizaje en el aula, y si han vencido el obstáculo de la integración académica (si en efecto había uno interpuesto específicamente en su camino) una vez matriculadas. Los resultados positivos son alentadores, pero no podemos afirmar que representan la vivencia material y generalizada a lo largo y ancho de las regiones apartadas de los centros urbanos. Hasta nos sorprenderíamos si fuera así por lo que sabemos de los graves problemas de corrupción e impunidad que sufre últimamente la instrucción pública en amplias franjas del sistema educativo, por ejemplo, en algunos estados de México ${ }^{8}$. En otros términos, hemos constatado en los tres estudios de caso que existe una correlación entre los datos reportados sobre la IPG por la UNESCO y la experiencia de las niñas respecto a su llegada a la escuela primaria rural. El siguiente objetivo de la investigación consiste en evaluar su experiencia en el aula: sentadas en el pupitre, medir el desempeño académico real. Luego, ¿cómo es la experiencia a su Ilegada a los niveles superiores? - en particular, el aprovechamiento en las asignaturas escolares más exigentes. De nuevo, la evidencia provendrá de la evaluación objetiva, externa e independiente.

8. En algunas regiones estatales, centradas precisamente en municipios rurales, las condiciones de trabajo más bien se acercan a las de una descomposición avanzada. Aquí es importante destacar que por los propósitos de la investigación educativa recomendamos no tomar en cuenta la información suministrada por las autoridades locales, ni intentar recoger datos de dichas comunidades en crisis. La recomendación que ofrecemos viene al caso de la propuesta que presentamos en la Conclusión. Esta triste circunstancia, como demuestran los resultados que hemos presentado, no corresponde a las escuelas donde han sido realizados los estudios de este informe. 
Tampoco es nuestra intención, en realidad sobra decirlo, relativizar la urgencia de aplicar medidas urgentes a las tareas de reforma educativa en esta materia. Pero para seguir avanzando es preciso efectuar un diagnóstico ajustado de los logros verificables hasta la fecha y una evaluación objetiva de: la capacidad actual del sistema, su capacidad potencial, las competencias pertinentes puestas en marcha sobre la tarea académica, y los poderes de convocatoria disponibles en los diferentes ámbitos, sociales y cognoscitivos, por parte de las mismas niñas escolarizadas. Luego, nos centramos en el remedio de los factores que limitan estas capacidades y competencias.

\section{Observaciones sobre los procedimientos}

Retomamos los resultados, inconclusos, de las entrevistas (por ejemplo la diferencia entre niños y niñas en la pregunta de la $E S$ ) en Tlaxcala que indagaron acerca de las percepciones afectivas. El riesgo (de sacar interpretaciones erróneas) radica en gran parte en la dificultad de controlar la manera en que los entrevistados entienden las preguntas. Como vimos, en las evaluaciones abiertas (que por cierto ofrecen grandes ventajas), debemos tomar en cuenta la intervención de factores no contemplados. Otra opción a la mano es dejar que las palabras de los informantes hablen por si mismas como en el estudio de Chiapas.

Reportamos otra aparente divergencia, en este caso entre entrevistados adultos, hombres y mujeres en un estudio realizado diez años después de las pruebas de lectura y escritura en Tlaxcala. Igual como en la $E S$, no se trató de una medida de habilidad lingüística o destreza de lectoescritura, sino de una opción estilística, por cierto ampliamente comentada en la comunidad: la alternancia entre las lenguas, en este caso, la frecuencia de tomar préstamo del español al narrar en náhuatl. Coloquialmente, se denomina "mezcla." Comparando cuentos adultos e infantiles, a diferencia de las niñas, las señoras se perfilaron menos "puristas" que los hombres (en la escuela, las niñas salieron igual de "puristas" como los niños). Los cuentos de las niñas, a diferencias de las señoras, salieron con el mismo índice de mezcla que pudimos marcar entre sus compañeros varones. De nuevo, la diferencia en el caso de las señoras resultó difícil de interpretar por la dificultad de controlar un factor externo en particular, entre hombres y mujeres: años de primaria cumplidos. La gran mayoría de los hombres habían terminado el sexto grado. Una minoría de sus vecinas lo había hecho. Por casualidad, el dato coincide con una observación que mencionamos en la sección anterior acerca de los cambios generacionales. Entre los señores jóvenes de hoy en día, haber cumplido los seis años de primaria se alza como logro de la gran mayoría, igual como es el caso para sus esposos y hermanos.

En cambio, las evaluaciones de lectura (Tlaxcala y Loja), menos abiertas que las entrevistas (y por lo mismo menos "ricas" en información sobre apreciaciones y posturas) no sufren en la misma medida del efecto de los factores impredecibles. Por ejemplo, en la prueba de lectura del sexto grado en Loja, una estimación de $r=.604$ en confiabilidad resultó aceptable, un índice importante, que en efecto se pudo estimar de una manera objetiva. Por estas consideraciones, de ninguna manera desfavorecemos un método sobre otro, sino a la hora de interpretar resultados tomamos en cuenta las limitaciones de cada uno. Para cada método, las limitaciones son diferentes (Fraenkel y Wallen, 2006). 


\section{ConClusión: UNA PROPUESTA PARA FUtUROS ESTUdios}

Para finalizar, los tres estudios de caso representan distintos contextos sociales: dos comunidades, una alta y la otra medianamente comunicadas, y una relativamente apartada. Pero no podemos tomar por hecho que los tres, en conjunto, forman una muestra representativa del medio rural. Tampoco podemos suponer, sin cuestionarlo, que todas las escuelas primarias rurales mexicanas y ecuatorianas cumplen con la norma de paridad (100 niñas para cada 100 niños) en la inscripción/asistencia. Mucho menos podemos descartar la posibilidad de una discrepancia general en destrezas de lectoescritura alcanzadas en las escuelas primarias rurales, con las niñas en desventaja, con base en nuestros resultados parciales. Así que proponemos la convocatoria de una investigación sobre la escuela primaria a escala regional centrada en los países con las concentraciones más importantes de populaciones no-urbanas: México y Centroamérica y los países andinos. Además, son los mismos países donde se concentra la población infantil de edad escolar, aprendices del español como segunda lengua. La primera meta de la investigación consistiría en comprobar:

(1) la paridad en inscripción/asistencia, reportada por los respectivos ministerios de educación, y

(2) una comparación favorable, en el desempeño real, en lectoescritura, tomando como hipótesis la que surgió del resultado de la evaluación en Tlaxcala y Loja, tomarla como predicción para aceptarla o rechazarla. Precisamente: que las calificaciones de las niñas salgan estadísticamente equivalentes, o superiores, en comparación con sus pares varones.

Se presenta como procedimiento necesario, por razones obvias, privilegiar la selección de comunidades apartadas y de escasos recursos materiales. Por las consideraciones metodológicas elementales de confiabilidad y validez, se aplicaría un instrumento sencillo del mismo tipo que se aplicó en Loja (y el mismo en cada sitio con fines de comparación) por un equipo externo de investigadores. Un requisito indispensable es desconfiar siempre de evaluaciones oficiales previas, estandarizadas o no-estandarizadas.

En todo análisis de los datos, tendremos la vista fija en la siguiente etapa escolar, la secundaria. ¿Qué tendencias e indicios sugieren condiciones propicias para mantener o mejorar la paridad (según el caso) después del sexto grado? Los métodos cualitativos, de observación y entrevista, resultarán indispensables como complemento de las pruebas. Tomando en cuenta la advertencia de la Nota 7, los resultados de una muestra de entre quince y veinte escuelas comunitarias podrían aportar un análisis decisivo para trazar los próximos pasos de la reforma del currículum educativo.

\section{REFERENCIAS BIBLIOGRÁFICAS}

Atal, J., Nopo, H. y Winder, N. (2009). New century, old disparities: Gender and ethnic wage gaps in Latin America. IDB Working Paper Series No.109. Washington DC: Inter-American Development Bank. 
Berlinski S., Galiani, S. y Gertler, P. (2009). The Effect of Pre-Primary Education on Primary School Performance. Journal of Public Economics, 93, 219-234.

Céspedes, C. y Robles, C. (2016). Niñas y adolescentes en América Latina y el Caribe: Deudas de igualdad. Santiago de Chile: Comisión Económica para América Latina y el Caribe.

Chanona Pérez, O. (2014). La niñas lacandonas de Lacanha Chansayab, Chiapas: el impacto de la alfabetización en su identidad. En J. M. Godínez Martínez, y B. G. Paredes Zepeda (eds.), Lengua multiculturalidad e identidad (pp. 145-170). México, DF: Fontamara.

Chiodi, F. y Bahamondes, M. (2002). Una escuela, diferentes culturas. Temuco: Corporación Nacional de Desarrollo Indígena.

Chireac, S.-M., Francis, N. y McClure, J. (2017a). Awareness of language: Literacy and second language learning of Spanish in Mexico. International Journal of Bilingual Education and Bilingualism, 20(3),1-14. http://doi.org/10.1080/13670050.2017. 1294143.

Chireac, S.-M., Francis, N., Guerrero-Jiménez, G., González Tamayo, N., Guamán, M., Martínez de Lara, A., Vacacela Medina, C. y Villalta Córdova, B. (2017b). Interculturalidad y educación bilingüe en la provincia de Loja. En A. D. Verdú Delgado y N. A. González Tamayo (Eds.), Conocimientos ancestrales y procesos de desarrollo. Nacionalidades indígenas del Ecuador (pp. 83-105). Loja: Universidad Técnica Particular de Loja. Recuperado de: http://dspace.utpl.edu.ec/ bitstream/20.500.11962/21489/1/Conocimientos\%20ancestrales\%20UTPL.pdf.

Díaz Montenegro, E. (2012). Una mirada a las contradicciones de la revitalización lingüística en El Cauca. Tabula Rasa, 17, 219-244.

Fraenkel, J. y Wallen, N. (2006). How to design and evaluate research in education. New York: McGraw Hill.

Francis, N. (2012). Bilingual competence and bilingual proficiency in child development. Cambridge: MIT Press. https://doi.org/10.7551/mitpress/9780262016391.001.0001.

Hall, G. y Patrinos, H. (2004). Indigenous peoples, poverty and human development in Latin America, 1994-2004. Washington DC: World Bank.

Hamel, R. E. (2008). Bilingual education for indigenous communities in Mexico. En J. Cummins y N. Hornberger (Eds.), Encyclopedia of language and education, Volume 5 (pp. 311-322). New York: Springer.

Hamel, R. E. (2009). La noción de calidad desde las variables de equidad, diversidad y participación en la educación bilingüe intercultural. Revista Guatemalteca de Educación, 1, 177-230.

Instituto Nacional de Estadística Geografía e Informática (INEGI) (1990). XI Censo general de población y vivienda (Tlaxcala). México, DF: INEGI.

Instituto Nacional de Estadística Geografía e Informática (INEGI) (2010). XIII Censo general de población y vivienda (Tlaxcala). México, DF: INEGI.

Instituto Nacional de la Mujer (INM) (2006). Las mujeres indígenas de México: Su contexto socioeconómico, demográfico y de salud. México, DF: INM.

King, K. (2001). Language revitalization processes and prospects: Quichua in the Ecuadorian Andes. Bristol: Multilingual Matters. 
Levy, D., Hausmann, R., Santos, M. A., Espinoza L. y Flores, M. (2016). ¿Por qué Chiapas es pobre? Cambridge: Center for International Development at Harvard University. Working Papers, N. 300.

Luna Ruiz, J. (2007). Nahuas de Tlaxcala. México DF: Comisión Nacional para el Desarrollo de los Pueblos Indígenas.

Meyers, M. (2004). African American Women and Violence: Gender, Race, and Class in the News. Critical Studies in Media Communication, 21(2), 95-118.

Nava Nava, R. (2014). Recursos para trabajar la literacidad en lengua náhuatl: Análisis de algunas experiencias y propuestas para su desarrollo. En J. M. Godínez Martínez, y B. G. Paredes Zepeda (Eds.), Lengua multiculturalidad e identidad (pp. 21-40). México, DF: Fontamara.

Nutini, H. y Issacs, B. (1974). Los pueblos de habla náhuatl de la región de Tlaxcala y Puebla. México, DF: Instituto Nacional Indigenista.

UNESCO (2012). Atlas mundial de la igualdad de género en la educación. Recuperado de: http://www.uis.unesco.org/Education/Documents/unesco-gender-educationatlas-2012-spa.pdf.

UNESCO (2015). Género y la EPT en el mundo, 2000-2015: Logros y desafíos. París: Organización de las Naciones Unidas para la Educación, la Ciencia y la Cultura (UNESCO).

UNICEF (2015). El aprendizaje bajo la lupa: Nuevas perspectivas para América Latina y el Caribe. Recuperado de: https://www.unicef.org/lac/UNICEF_Aprendizaje_ bajo_la_lupa_nov2015(1).pdf.

Unterhalter, E., North, A., Arnot, M., Lloyd, C., Moletsane, L., Murphy-Graham, E., Parkes, J. y Saito. M. (2014). Análisis de investigaciones rigurosas sobre educación. La educación de las niñas y las jóvenes y la igualdad de género. Recuperado de: http:// www.unesco.org/fileadmin/MULTIMEDIA/FIELD/Mexico/informeninas_01.pdf.

Vezzali, F. (2016). Adolescentes y jóvenes fuera de la escuela: Las demandas para una enseñanza secundaria garante de derechos en América Latina y el Caribe. São Paulo: Campaña Latinoamericana por el Derecho a la Educación. 